\title{
Serine/Threonine-Protein Kinase PLK4
}

National Cancer Institute

\section{Source}

National Cancer Institute. Serine/Threonine-Protein Kinase PLK4. NCI Thesaurus. Code C119603.

Serine/threonine-protein kinase PLK4 (970 aa, 109 kDa) is encoded by the human PLK4 gene. This protein plays a role in the duplication of centrioles during the cell cycle. 